\title{
Designing Shared Public Display Networks - Implications from Today's Paper-Based Notice Areas
}

\author{
Florian Alt ${ }^{1}$, Nemanja Memarovic ${ }^{2}$, Ivan Elhart ${ }^{2}$, Dominik Bial ${ }^{1}$, Albrecht \\ Schmidt $^{4}$, Marc Langheinrich ${ }^{2}$, Gunnar Harboe ${ }^{3}$, Elaine Huang ${ }^{3}$, Marcello P. Scipioni ${ }^{2}$ \\ ${ }^{1}$ University of Duisburg-Essen, Schützenbahn 70, 45117 Essen, Germany \\ \{florian.alt,dominik.bial\}@uni-due.de \\ ${ }^{2}$ University of Lugano, Via G. Buffi 13, 6904 Lugano, Switzerland \\ \{ivan.elhart, marc.langheinrich, nemanja.memarovic, marcello.scipioni\}@usi.ch \\ ${ }^{3}$ University of Zürich, Binzmühlestrasse 14,8050 Zürich, Switzerland \\ \{harboe, huang\}@ifi.uzh.ch \\ ${ }^{4}$ University of Stuttgart, Pfaffenwaldring, 70569 Stuttgart, Germany \\ albrecht.schmidt@acm.org
}

\begin{abstract}
Large public displays have become a regular conceptual element in many shops and businesses, where they advertise products or highlight upcoming events. In our work, we are interested in exploring how these isolated display solutions can be interconnected to form a single large network of public displays, thus supporting novel forms of sharing access to display real estate. In order to explore the feasibility of this vision, we investigated today's practices surrounding shared notice areas, i.e. places where customers and visitors can put up event posters and classifieds, such as shop windows or notice boards. In particular, we looked at the content posted to such areas, the means for sharing it (i.e., forms of content control), and the reason for providing the shared notice area. Based on two-week long photo logs and a number of in-depth interviews with providers of such notice areas, we provide a systematic assessment of factors that inhibit or promote the shared use of public display space, ultimately leading to a set of concrete design implication for providing future digital versions of such public notice areas in the form of networked public displays.
\end{abstract}

Keywords: public display, observation, advertising

\section{Introduction}

Large digital displays are rapidly permeating public spaces. The availability of suitable display technologies for outdoor use, together with decreasing prices for large screen display hardware, has led to a transformation from paper-based to digital signage and advertising. Urban landscapes are being augmented with digital signage solutions by large digital-out-of-home (DOOH) advertisers (e.g. Stroer, JC Decaux) replacing more and more traditional billboards. Apart from reducing the cost of updating their contents, these displays allow for the addition of animations and/or interlacing news content in order to make them more visible and attractive to passers-by. 
However, so far these digital displays are not globally networked and access is typically restricted to their owners.

We envision that in the future, these individual displays and isolated display solutions could be inter-connected through the Internet. Hence, a canvas across urban space can be provided that allows for distributing any type of content onto this display landscape, not only from large advertisers but instead from neighboring shops, local residents, and visitors. Technically, the challenge of such a vision is to create a suitable middleware that supports the remote exchange and programming of arbitrary content onto arbitrary displays, as well as suitable interfaces to interact with such systems. A far greater challenge, though, lies in the design and deployment of suitable control tools that can support the stakeholders' understanding of how these displays ought to be used. Without suitable incentives and means for staying in control, display owners might be reluctant to grant access to their displays and relinquish their control over what is being shown on their in-store displays. Our central research question thus is: how can we build digital public display networks that can go beyond today's isolated advertisement displays, and instead provide an open platform for posting and displaying user-generated content? Yet instead of tackling the technical challenge of such a vision directly, we begin our investigation with trying to understand the social and economical drivers to support this vision: What would motivate display owners to allow others access to their displays? And what would control interfaces and incentive structures have to look like to support widespread uptake of such systems?

We decided to ground our research in today's practices surrounding the precursors to our vision: shop-windows, notice boards, and wall hangers, where customers, community members, and visitors can use pen $\&$ paper and pins $\&$ tape to put up their messages, notices, posters, and classifieds (c.f. Figure 1). For the purpose of this paper, we collectively call such boards, walls, and hangers "Public Notice Areas", or PNAs. We began by observing the use of PNAs in 29 locations in two different countries, using photo logs to document their use and change over 4 weeks. We then performed in-depth interviews with most of the people responsible for the observed PNAs, in order to understand their current practices for controlling access to the PNAs, elicit their motivations for offering PNAs, and identify concerns they might have when relinquishing control in a fully digital public display network. The results from the observational studies and interviews were analyzed in a quantitative and qualitative fashion, using a "data walkthrough" analysis that we developed to give all team members an in-depth view of the data collected by other team members.
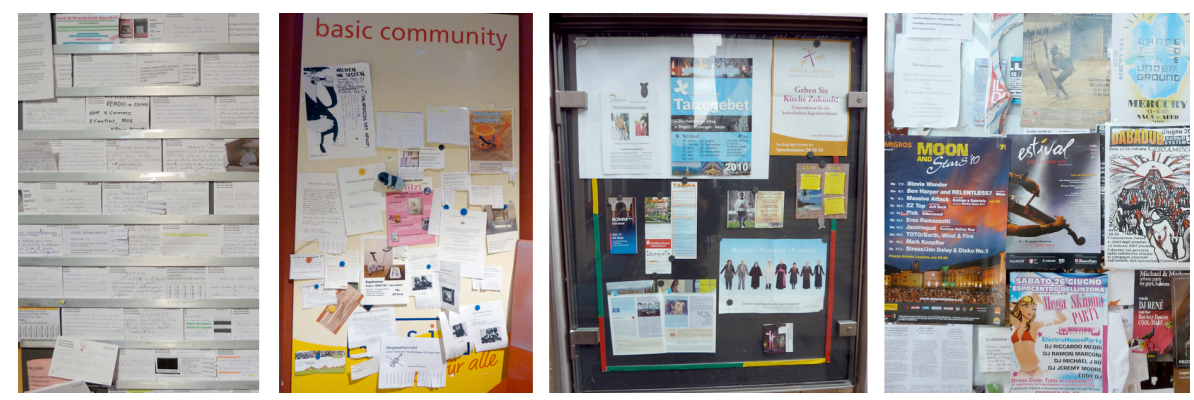

Fig. 1. Types of Displays. (1) Scaffolded Classifieds Display (2) Non-Scaffolded Classifieds Display (3) Information Display (4) Event Display 
After briefly discussing related work in section 2 below, we will enumerate our research questions in section 3 , followed by a description of our study design in section 4. Section 5 describes the data analysis and section 6 presents our findings based on the research questions. Finally section 7 deals with inferred design implications.

\section{Related Work}

Public displays have been subject to research for many years. With decreasing prices, many traditional displays are being replaced with digital counterparts. Many projects have looked at the technical requirements for networking digital displays, mostly within and across offices (e.g. [1], [3]) but also in public space (e.g. [12],[15]). Also, novel interaction methods have been studied, both in terms of user behaviors (e.g. [2],[11]) and interface technology (e.g. [6],[7]). Although technical and architectural suggestions could be drawn from these studies, our initial work in this domain focuses on understanding the design implications from existing practices of posting on PNAs and latent motivations for offering and maintaining PNAs.

There have been several such studies that looked at current practices around publicly available notice boards and displays. Taylor et al. [16] looked at community notice boards in a rural village to inform the design of a digital version. Churchill et al. [4] looked at community notice boards in an urban area and in their own workspace to inform the design of their Plasma Poster Network, a system that enhances the chance to encounter interaction and awareness of different workgroups' activities. Huang et al. [8] conducted a field study to analyze various paper and digital displays and their actual placement, as well as how much people actually look at them. Based on their findings, they provided design recommendations for increasing the visibility of displays and for better matching between people's behavior and the displays' content. Although there is some overlap between previous studies and our research, our primary goal is to come up with general implications that inform the design of a public display network system that go beyond display visibility and office space and suburban area settings.

As can be seen from the previous paragraph, public displays have a large potential to foster communities. Redhead et al. [13] presented a qualitative analysis of local community interaction among its members. One of their main findings was that public displays could increase the perception of unity as well as communities' interests. Some of their findings, e.g. suggestions for content and features of such displays, support the perspective of the community and delivery of local messages. A report of their findings on the usage of a digital community notice board is available in [14].

Studies exploring the impact of digital notice boards on communities have been conducted in several settings. As mentioned previously, Taylor et al. [17] looked into notice board practices in a rural village and informed the design of the Wray Display, a community photo sharing display aimed at understanding how digital displays can help to support communities in suburban areas. Churchill et al.'s Plasma Poster Network [5] looked into how displaying social media impacts relationships among colocated colleagues in their workplace. The CoCollage [10] aimed at cultivating community in a café by showing posters and quotes and hence enhancing awareness, in- 
teraction, and relationship among people. Of particular interest is Huang et al.'s [9] finding that people spend less time learning about system capabilities when it is not supporting current use practices. The users' desire and interest to use novel system need be taken into account [11]. This emphasizes the necessity of embedding existing routines in novel system to support its use. Note that in our view, this entails not only catering to users' needs, but also reflecting on PNA providers' motivations.

\section{Sharing Public Display Space}

Traditional public displays are a very common way of communication and they are ubiquitous in our environment. They scale from post-it notes on an office door telling people who stop by "back in 5 minutes" to graffiti on a train making a political statement. This form of communication is very effective and observers will not be even aware of reading the signs in many cases, but they still do. Such public displays are an example of invisible technologies that allow transparent use, as Weiser suggests [18].

In our work we are particularly interested in public displays for information dissemination and for one-to-many communication. Spaces for these forms of communication and publicly sharing information can be found in many places, such as grocery stores, cafes, and restaurants but also in city administrations, public libraries, universities, and schools. Such places provide space that is visible, accessible, and frequented by people. Examples are notice boards and walls on which people are allowed to attach posters, and windows as well as doors where people can hang up flyers or notes. There is a huge variety of such PNAs, and many types of content can be found. Generally it can be seen that these displays have a function in their environment, and that the form factor of the display and the types of contents shared are influenced by the location, the owner, and the expected audience. In contrast to other forms of communication these displays support the following properties: (1) dissemination of content that is mainly locally relevant, (2) addressing of the receivers by selection of space, and (3) forcing information and content on people that pass through a certain space.

Traditionally, posting information in PNAs also had the function of personal communication from one individual to many receivers. However, this function of public displays has lost importance with the digital social networks and the World Wide Web. Popular forms of content include sales, housing, job and service offers, events, promotions, lost and found, and advertisements, all of which are at least showing one of the characteristics above. In order to derive an understanding of how to create digital displays that provide new flexibility and cost-effectiveness and at the same time retain the qualities of the analog PNAs, we investigate several issues further.

\subsection{Value Propositions of PNAs for Stakeholders}

In the optimal case, PNAs provide value to all stakeholders, including the people owning the space, people providing content, and people observing the content. First, it is important to identify stakeholders for a PNA installation, and their motivations. In many cases there is interplay between interests, incentives, and value propositions. 
Consider the following scenario of a notice board for classified ads in a supermarket. The content provider (e.g. a customer who wants to sell a bike) has the opportunity to reach people in the local community. The observer (e.g. another customer looking for a used bike to buy) via PNA becomes aware of the product she is looking for. The supermarket provides customers with a further reason to visit the store.

Values have to be seen in the greater context of the PNA and its place of use. Here issues such as exercising control over what content can be placed, by defining access to the display and implementing means for restricting content placement to a certain group play a role. The following questions help to identify these issues:

- Who is allowed to post?

- Who decides what content is appropriate?

- What content can be posted?

- What is the motivation for the owner to allow posting?

- What is the motivation for content providers to post?

- What is the motivation for observers to look at the content?

\subsection{Mechanism for Content Creation, Posting, Removing and Access Control}

The utility of public displays depends on the fact that displayed information is useful. In general, content is posted to the display and removed or overwritten after a certain time. The mechanisms used both for posting and removing content as well as for enforcing usage policies are essential success factors. Often it is desirable that content can be created ad-hoc, without specific knowledge or tools (e.g. writing with a pen provided at the board onto pre-printed cards). Yet, the simpler the means for content provision, the more likely that spam or inappropriate content will be posted.

Many different ways can be found with which such control mechanisms for content creation and posting are restricted, ranging from having the notice board in a public area (where users posting content may be observed) to explicit approval of content. We suggest the following questions to identify and structure these mechanisms:

- How can content be placed onto the display?

- What flexibility is available for content creation?

- What tools are required to create content?

- What is the process for approval of content?

- How is content removed or overwritten?

- How is access control implemented for content providers and viewers?

- How are viewers supported to help them remember content?

\subsection{Learning from Practices in the Analog World}

Many different types of PNAs are in use in different places. This multitude evolved over a long time and many of their properties fulfill a certain need. Similarly, many sorts of posted content are publicly posted. Here, too, a long tradition exists for creating and designing content, ranging from artistic expression to minimalistic presentations. In this paper we aim at identifying these rich characters of different displays, content types, and related practices. For deriving design implications for digital dis- 
plays, understanding practices and the rationale behind these is very valuable. In particular we are interested in the communication aspects that such displays facilitate.

A further important aspect of public displays is that they have a potential function for the community. By positing information in publicly accessible space but in a specific location, a clear addressing to the local community is made. Here it seems interesting to uncover functions that displays have that go beyond the communication of individuals or groups. To learn about the practices we ask the following questions:

- What practices have been established around sharing on a display?

- What are the reasons for these practices?

\section{Study Design}

To answer our research questions we ran a two-week field study during summer 2010 in four different cities in Switzerland and Germany, involving observational studies (photo $\operatorname{logs}$ ) and subsequent interviews with the people responsible for the observed PNA, i.e. shop-owners and personnel.

\subsection{Observational Studies}

We aimed at observing a wide variety of locations, displays, and audiences. To do so, we looked for any kind of institutions, stores, and restaurants/eateries that displayed public notice boards. Due to the labor-intensive nature of the work, we opted for a convenience sampling of the observed sites (places were located along our work routes), allowing us to regularly visit these places over the course of four weeks. Consequently, the observed locations were within the local neighborhoods surrounding the universities and central stations of Lugano (Switzerland), Essen, Düsseldorf and Munich (all Germany). An overview of the locations can be found in Table 1.

After choosing suitable locations, we identified the persons responsible for the PNA to be observed. We introduced ourselves, explained the purpose of the study and asked for permission to take pictures of the PNA. We provided a written description of the study and explained that all data collected would be used for scientific purpose only. While most people immediately agreed to permit the study and even showed interest in the results, some of them (notably in larger stores) first had to check with central management and asked us to report to the management every time we returned. In two locations we were refused permission to conduct the study, as the management felt that this would strongly affect their customers' privacy.

After permissions had been obtained, we visited each location on consecutive working days over the course of roughly four weeks, each time taking several pictures of all postings. Pictures were mainly taken in the morning (on the way to work), during lunch break, and in the late afternoon / evening (on the way back home). We tried to make sure that pictures were taken at comparable times of the day. In total, 4 researchers were involved in the study, each one being assigned a fixed set of locations. Due to scheduling constraints it was not in all cases possible to take pictures on 
consecutive working days. However, we made sure that for each location at least 10 picture sets from different days were taken within no more than 4 weeks.

Table 1. Overview of study locations.

\begin{tabular}{|c|c|c|c|c|c|}
\hline ID & Name & Description & Obs. I Int. & Type & Cur. \\
\hline E1 & Turn Headshop (Rack) & Retail & $\mathrm{x} \mid \mathrm{x}$ & ED & - \\
\hline E2 & Turn Headshop (Door) & Retail & $\mathrm{x} \mid \mathrm{x}$ & ED & - \\
\hline E3 & Diocese (Office) & Church & $\mathrm{x} \mid \mathrm{x}$ & ID & $\mathrm{x}$ \\
\hline E4 & Diocese (Entrance) & Church & $\mathrm{x} \mid \mathrm{x}$ & ID & $\mathrm{x}$ \\
\hline E5 & Supermarket & Retail & $\mathrm{x} \mid \mathrm{x}$ & $\mathrm{SCD}$ & - \\
\hline E6 & Supermarket & Retail & $\mathrm{x} \mid \mathrm{x}$ & UCD & - \\
\hline E7 & University cafeteria & Public Bldg./Gov. & $-1 x$ & UCD & $\mathrm{x}$ \\
\hline D1 & City administration & Public Bldg./Gov. & $\mathrm{x} \mid \mathrm{x}$ & ID & $\mathrm{x}$ \\
\hline $\mathrm{D} 2$ & Adult Education Center & Public Bldg./Gov. & $\mathrm{x} \mid \mathrm{x}$ & UCD & - \\
\hline D3 & Public Library & Public Bldg./Gov. & $x \mid x$ & ED & $\mathrm{x}$ \\
\hline $\mathrm{D} 4$ & Child Services & Public Bldg./Gov. & $\mathrm{x} \mid \mathrm{x}$ & ED & - \\
\hline M1 & Supermarket & Retail & $-1 x$ & SCD & - \\
\hline $\mathrm{M} 2$ & Supermarket & Retail & $-1 x$ & SCD & - \\
\hline M3 & Supermarket & Retail & $-1 x$ & SCD & - \\
\hline M4 & Supermarket & Retail & $-1 x$ & SCD & - \\
\hline $\mathrm{L} 1$ & Supermarket & Retail & $x \mid x$ & SCD & - \\
\hline $\mathrm{L} 2$ & University & Public Bldg./Gov. & $\mathrm{x} \mid \mathrm{x}$ & $\mathrm{UCD}$ & - \\
\hline L3 & Bakery & Service & $\mathrm{x} \mid \mathrm{x}$ & UCD & $\mathrm{x}$ \\
\hline L4 & Church & Church & $x \mid-$ & ID & $\mathrm{x}$ \\
\hline L5 & Supermarket & Retail & $\mathrm{x} \mid \mathrm{x}$ & SCD & - \\
\hline L6 & Café & Service & $\mathrm{x} \mid-$ & ED & - \\
\hline L7 & Hairdresser & Service & $x \mid-$ & ED & - \\
\hline L8 & Bar & Service & $\mathrm{x} \mid \mathrm{x}$ & ED & - \\
\hline L9 & Café & Service & $\mathrm{x} \mid \mathrm{x}$ & ED & $\mathrm{x}$ \\
\hline $\mathrm{L} 10$ & Pharmacy & Retail & $\mathrm{x} \mid-$ & ED & - \\
\hline L11 & Bookstore & Retail & $\mathrm{x} \mid \mathrm{x}$ & ED & - \\
\hline $\mathrm{L} 12$ & Red Cross & Public Bldg./Gov. & $\mathrm{x} \mid \mathrm{x}$ & ID & $\mathrm{x}$ \\
\hline L13 & Laundry & Service & $\mathrm{x} \mid-$ & ED & - \\
\hline L14 & Church & Church & $\mathrm{x} \mid-$ & ID & $\mathrm{x}$ \\
\hline \multicolumn{2}{|c|}{$\begin{array}{l}\text { Abbreviations: } \\
\text { Obs I Int: Observation I Interview } \\
\text { Cur: Curated Display }\end{array}$} & $\begin{array}{l}\text { Display Types: } \\
\text { SCD / UCD (Scaffolc } \\
\text { play), ID (Informatio }\end{array}$ & Display), ED & ent Disp & \\
\hline
\end{tabular}

\subsection{Interviews}

After finishing our observational study we conducted a number of interviews with people in charge of managing the displays. Those were not necessarily the display owners, but also store managers or regular staff. With the interviews we aimed at understanding a range of issues surrounding PNAs: the shops' motivation for having such a PNA; the practices for adding, editing, and removing content; any restrictions 
as to what customer were allowed to post; any problems with the displays; and whether people could imagine substituting the "analog" display with a digital version.

We conducted interviews in the locations that were covered in our observational study. However, for two locations we were not able to get hold of a responsible person. We also included additional locations with similar PNAs to gather further information. For the interviews we returned to the location and tried to identify the person (currently) in charge of the display, asking her or him to answer a set of 10 questions. We offered to return at a convenient time in case people were too busy to talk to us. As interviews happened during business hours, the interviewee's time was in general scarce. Consequently, we limited our interviews to maximum 10 minutes. We either audio-recorded the interviews with a voice recorder (in case people felt comfortable to do so) and transcribed them later, or took hand-written notes during the interview. It should be noted that the interviews were limited to the parties who owned and "administered" the displays for reasons of accessibility. Information we gained about the perception and use of these displays by passers-by and other stakeholders in the interviews was conveyed to us by display administrators and therefore may reflect their particular interpretation of phenomena regarding the displays. For the purpose of this study, we relied primarily on our observations to gain insight into the practices and needs of passers-by and other stakeholders to complement the more direct inquiry into the practices of display owners and administrators.

\section{Data Analysis}

We conducted an extensive qualitative analysis of the collected photographs and interviews. Because of the large volume of data generated by the study and the distributed fashion in which the data was collected, we designed a "data walkthrough" analysis method. The goal of this method was to help team members become highly familiar with data that had been collected by other members of the team, and provide a view on that data that would afford both a comprehensive overview of all of the data from all of the sites, as well as an individual detailed view of each site.

The team printed out photos from each day of data collection for each data collection site and affixed these photos (in total 298) to walls and whiteboards in a single room. The photos were placed in chronological order, grouped by site. Additionally, interview transcripts and field notes were affixed along with the corresponding photographs. Five members of the research team then proceeded to "walk through" the data, analyzing the photographs, interviews, and notes, and writing observations on individual sticky notes that were used to annotate the collections of data (see Figure 2 ). As the team discovered patterns and higher-level observations, these were written on a separate whiteboard.

After this exploratory phase of analysis, the team then used an affinity diagram to identify themes in the data and associate them with our research questions (see (3) Affinity Diagram in Figure 2):

- Who are the stakeholders? (6.1)

- What "characters" of displays arise and what determines them? (6.2)

- What are current practices for sharing displays space? (6.3) 
- What is the role of "posting displays" in a space? (6.4)

- How do the space, stakeholders and content interplay? (6.5)

- What are the needs of people who are posting and display owners, and how do the displays satisfy them? (6.6)

This was achieved by taking all of the observations and categorizing them to derive the general findings from the data. The identification of findings was done as a group, and each observation was discussed as to how it might fit with other observations.
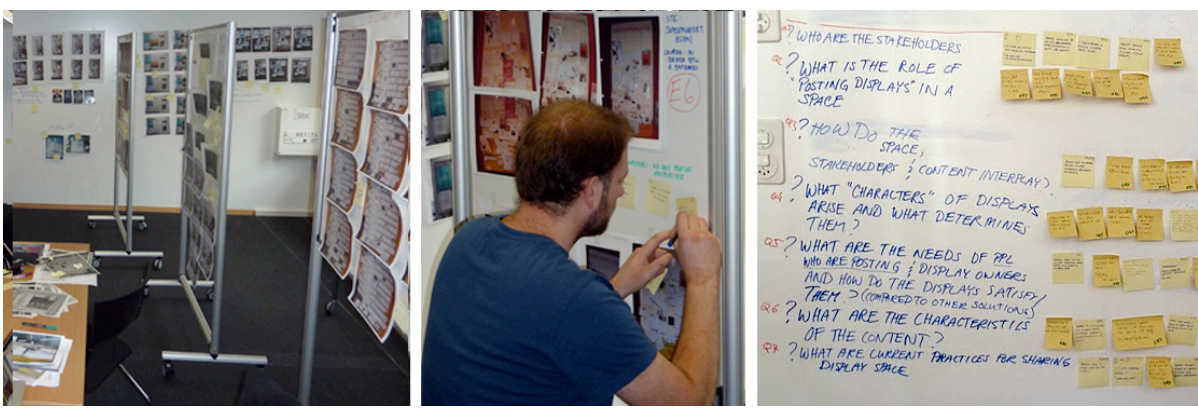

Fig. 2. Data Walkthrough (1) Photos, field notes, and transcribed interviews were printed and affixed to walls. (2) Analysis and annotation of material by researchers.

(3) Affinity Diagram to identify themes in the data.

\section{Findings}

Based on the data collected and analyzed, we report in this section the findings, with a focus on current practices. We first identify stakeholders, describe characteristics of displays and content, and uncover the motivation for shared public displays. During the data analysis we did not discover any obvious differences in the data gathered in both countries.

\subsection{Stakeholders and Motivation}

The data from observations and interviews provide a clear indication of a number of diverse stakeholders involved in operating and using public notice boards and shared public displays. On a highest level we can discriminate three different groups: display providers and managers, content providers, and viewers.

\section{Display Providers and Managers}

Based on interviews (L1, L2, L5, E3, E4, E5, E6, D1, M1, M2, M3, M4) we discovered that the decision to install a PNA is taken on a higher management level, e.g. in store chains and public authority institutions, and hence each branch or store will have a PNA as standard inventory. Also public and ecclesiastic institutions see information dissemination as a part of their mission and use them to distribute important informa- 
tion about their current activities. In locations where venue and shop owners are running the place (L3, L8, L9, L11, E3, E4, E5, M1, M2, M3, M4), interviews revealed that the decision to have a display and how to use it is in one hand.

The motivation for providing public displays is manifold: retail and service have them to increase customer satisfaction (interviews at M1, M3, M4, E6, D2), public authorities and ecclesiastic institutions mainly used them to disseminate information on their current activities (observations at L4, L14, interviews at E7, D1, D2, D3, D4), and some of them (interviews at E3, E4, E7, D1, D2, D3, D4) feel the need to have a space for third party content as long as it fits within the institution's scope and does not harm its reputation.

In interviews, we found that some venues (L3, E3, E4, M3) have a dedicated person in charge of the content approval, i.e., a notice board manager whereas in other places it is less formalized. In the case of public and ecclesiastic institutions there is typically a dedicated manager, whereas smaller venues, like shops and cafés are more likely to distribute this role throughout their staff, i.e., each staff member can act as a manager.

\section{Content Providers}

We can see two distinct groups of content providers: classifieds providers and third party advertisers. Both groups seek to distribute information to the target audience. People living in the vicinity of the venue or its frequent visitors can be seen as classifieds providers, seeking for 'matchmaking' opportunities, e.g. students exchanging books, people offering and looking for housing, or selling furniture. The content defines in many cases how long one can expect a poster to remain on the board.

While classifieds providers are mostly individuals, third party advertisers are usually affiliations: church, government, business, musicians, non-profit, or other. All of them have a common goal of reaching a large audience and advertise in the vicinity of the target community's physical center, e.g. music events have multiple posters at music-oriented bars and universities, church-related events appear within its parish's locality, and even third party advertisements on government public display are topically focused. Interestingly enough, some of the venues take on the role of the third party advertiser and try to blend in with the rest when advertising its own events (e.g., Li8).

There is an inherent tension between display owners and content providers as both rely upon each other (e.g. a PNA without content is not interesting and a person providing content cannot do so without space). The best way to minimize the risk of conflicts is to create a shared understanding about venues' board expectations (e.g. it is clear what content is expected on a certain display). It seems that this is quite common for PNAs as there were very few reports of abuse of the displays (interviews at L1, L5, L8, L9, L11, E1, E2, D1, D2, D3, D4).

\section{Viewers}

The motivations for viewing content ranges from clear information needs (e.g. someone looks actively for a place to rent among postings) to accidental reading (e.g. waiting at the bus shelter and reading the posts in lack of any other occupation). Viewers 
are typically related to the location (e.g. they work or live close by) and may act at some time as viewer and at some other times as content provider.

Many PNAs are located near high-traffic areas with guaranteed waiting time, e.g. next to printers and copiers, whereas other locations use them for decoration, e.g. bars. In cases where people are waiting, e.g. for a drink or a print job to finish, it is very likely that they browse through the PNA's content. Supporting evidence can be found in [5].

\subsection{Displays and Content}

During our observations and data analysis we discovered a number of different display types that are targeted to specific types of content. In the following we discuss typical groups that are commonly in use for PNAs (see also Figure 1).

Scaffolded Classifieds Displays: Our observations indicate that retail stores and supermarkets favor a well-organized arrangement of their PNA (e.g., L1, L5). These areas are highly scaffolded with preprinted cards provided at the display, which can be filled in and inserted into several rows of slots. Their content is in general informal and hand-written and sometimes includes tear-aways (e.g. name and telephone number). Content creation is very fast (on the order of a minute). Typically, content providers are asked to provide a date to later remove outdated content. Often content not fitting the scaffold is attached next to the board. Content usually has a high turnover.

Unscaffolded Classifieds Display: These displays are characterized by the absence of prescribed structure leading to flexible and ad-hoc posting practices. Typically they are not well organized. Content in any form can be placed at any position, even if it fully or partially occludes other content. Interviews showed that for most of these displays (e.g., L2, L8), there is no particular person in charge to check and remove posters placed in improper place or with unwanted content. These displays reflect the selfservice nature of the postings. Content posted on such displays is in general similar to the aforementioned displays, with less structured layout, mixed sizes of posts, more colorful posts and more event-related content. We discovered such displays at university, the adult education center, and also grocery stores (L2, L8, D2).

Information Display: As part of their information duty, many institutions, churches and libraries provide curated PNAs (observations at L4, L12, L14). They are characterized by formal, mostly professional content, including ads and events. In general they have a smaller number of postings compared to the abovementioned types. Content is thematically focused (even if from third parties) and often applies to a larger vicinity. There is often a process for submitting and approving through existing/formalized organization networks. These PNAs typically have a means for prohibiting unauthorized postings (e.g. by having a glass front pane, see Figure 1c).

Event Display: Observations showed that bars and retailers offer event-focused PNAs (E1, L6, L8, L9). They are characterized by professional ads (posters, flyers), are thematically tightly scoped (e.g. techno events) and contain mostly third party content. The content is usually colorful, sometimes chaotic (depending on whether scaffolding is provided) and often provides some form of urban aesthetic. 

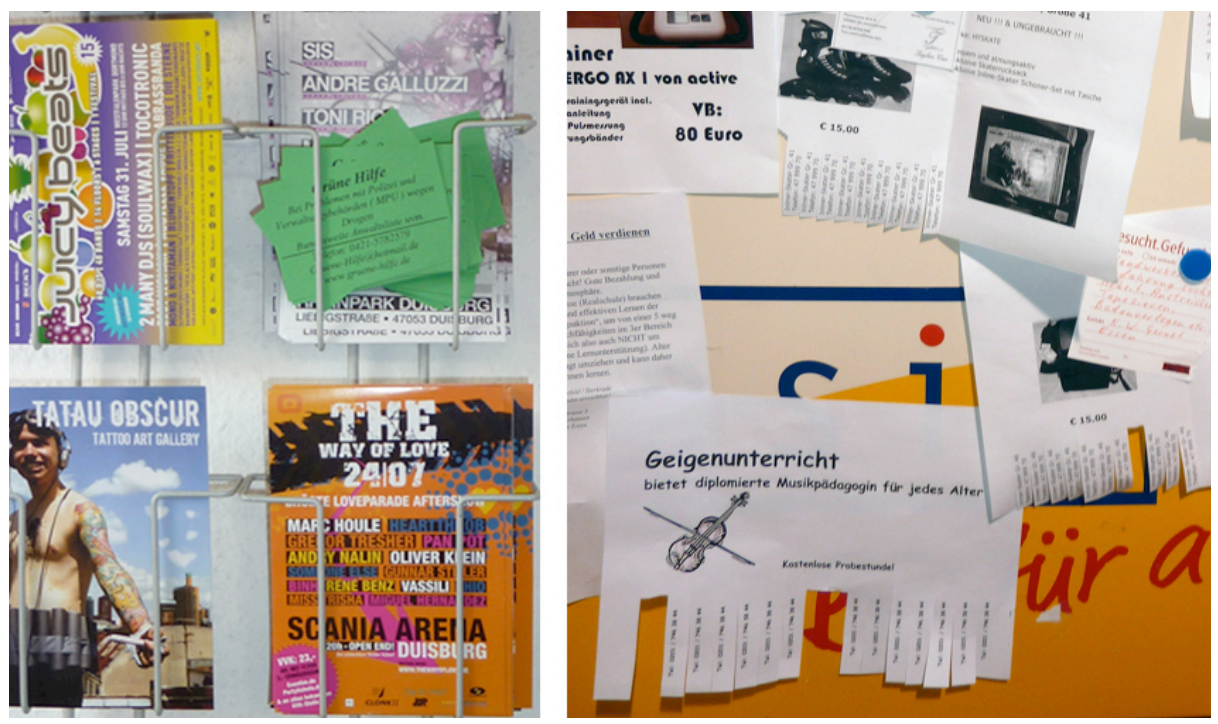

Fig. 3. Taking away information - Flyers (left, E1), Posts with takeaway tabs (right, E6).

\subsection{Managing Content and Supporting Memory}

Different mechanisms exist that help viewers to remember content they have seen. We came across 3 types of practices with regard to supporting memory: (1) information that is meant to stay on the board and where viewers are expected to remember essential information, (2) content that is completely taken away, and (3) content that offers parts with contact details that can be taken away. To allow for taking away information, current practices include providing multiple copies (e.g. flyers) or posts with takeaway tabs (see Figure 3). If viewers have to remember content, it is important that this is well feasible, e.g. by providing an easy-to-remember URL. With the wide availability of mobile-phone cameras we also see a further practice conceptually combining (1) and (3). As people take photos of public display content, they take the information with them and at the same time leave the content for others.

We found different practices of post management with regard to cleaning displays.

- Expiration Date: The most popular practice we discovered for cleaning content is the removal of outdated content. This can be done either with a posted expiration date (e.g. after 30 days of posting) or with an implicit expiration date (e.g. the date of an event).

- Complete Cleanup: Another practice is entirely erasing PNAs based on a regular schedule, e.g. monthly. We found this practice for PNAs that contained too much content for manually selecting stale content (observation and interview at L2).

- Curated Content: Especially in municipal and official institutions we found that displays are often curated. Content is usually submitted at the reception or sent via mail, and has to be approved by the director prior to being published. 
We observed that curated displays are in general very tidy and posts are not attached above each other so that they obscure other content.

\subsection{The Role of Shared Displays in a Space}

One function of displays is that they tend to create a central location for community activity (observations at E1, E2, E7). Such communities may be geographical, cultural, and also religious. Even though platforms such as Ebay or Craig's list exist, means for locally exchanging goods are still of interest. Especially items whose shipping costs outweigh their price are popular. Notice boards offer a convenient way of offering items to a local community where it is likely to find interested persons who are able to fetch items personally. Additionally, we found content that is relevant to the expected community visiting the location. E.g. in a music bar (observation at L8), PNAs mainly included promotion for music events (parties, live shows, concerts, etc.).

Further, boards are often used as community support tools. An example is the PNA at the adult education center, which was mainly used as a forum to exchange studyrelated material (e.g. people offered their course material to people in lower courses).

\subsection{Interplay between Space, Stakeholders, and Content}

We found that PNAs are often placed in high traffic areas (e.g. next to the main entrance of a location or in spots with waiting times) and that this had a strong impact on turnover in content. The same appeared to be true for communication and information hotspots. PNAs were often placed next to opinion boxes, store hours, space for prospects, and content from the display owner itself.

Most interesting though, posts are very local in nature. We found that content with no relation to a certain location (e.g. products, movies) appeared only rarely. In contrast, content seemed to be locally highly relevant. An example is posts on babysitting, as these people would usually not cover too large distances. This finding is also supported by the fact that information on local events is often posted in multiple locations in close proximity (e.g. in various stores in the pedestrian area). Similarly, boards often seem to express the identity of a venue. During our interviews we found that certain types of content is removed (e.g. political content, certain music events).

\subsection{Needs of Content Providers and Display Owners}

We observed that PNA owners often have an agenda. This in general correlates with their motivation for having a PNA (e.g. information obligation, dissemination of culture). Interestingly, persons in charge of managing the PNA sometimes serve as a gatekeeper, hence supporting certain events in an altruistic way as they feel that competing events are already sufficiently well publicized. As an example, the public library D4 refuses to announce Mardi Gras events but instead favors independent theatres. 
Further, many places provide support for posting. Such support includes tables for writing posts as well as scaffolding in the form of structured cards and pens.

In addition, we found that flexibility of content creation is supported in many places. Whereas especially scaffolded displays provide standard cards, most of the display owners allow in parallel for customized postings. Thus, homemade posts, professionally printed or colored to make them more visible and eye-catching, can also be found. Another example we observed was that it seemed to be OK for most display owners to extend the provided display space in order to fit more content, e.g. by attaching content next to the designated posting area.

We also found many places that allow for multiple copies of the same item of content. This was observed for different types of PNAs. On unscaffolded displays posters are freely tiled up next to each other, making a larger area more distinguishable from other parts of the board. This practice was observed also with scaffolded displays. Multiple posts of the same ad/post appear on different PNA locations and sometimes with small or no differences that are not easy spotted: in cases of non-professional handwritten posts, multiple posts are hard to recognize. Only by reading them carefully and comparing contact details can these be noticed. Obviously, having multiple copies of a single post increases the chances of the post being seen. The motivation for the content providers to put up multiple copies of the post is that they are afraid that their post is going to be removed for some reason.

Finally, we found that there are often difficulties of indicating a venue's expectations of the board. When asking for problems with PNAs, several display owners reported on discussions with people in order to explain what content is / isn't allowed. Content that is removed is mainly political content, offending or provocative content, competing content, and content that does not fit the agenda of the display owner. There are very few examples where we found explicit notices that certain types of content are not allowed or have to be approved. One example was at the adult education center, where a notice stated that teaching content is to be approved first.

\section{Design Implications}

Our observations identified important stakeholders and some of their needs, as well as current processes and structures helping them to fulfill their goals, or that emerge as a result of their joint activities. Naturally traditional displays are not technically networked. However we could observe a connectedness on a conceptual level. Connectedness was exhibited by enforcing the same policies across a set of displays or by having the same design and structure across all displays. Our design implications assume that the stakeholders' needs will still have to be met within an open digital public display network, but that the concrete processes and features do not need to match existing solutions one-to-one. We propose 5 broad principles, and offer specific ideas for how to apply them. 


\section{A. Design for Specific Uses of Notice Boards}

One of the clearest patterns in the observations was the great variety of notice boards and surfaces, depending on the purpose they were meant to fulfill, particularly the type of content they present, and on the preferences of the stakeholders. This indicates that no single design may be an appropriate replacement for all the current uses of notice boards. A system that displays large numbers of classified ads should optimally look different and work differently from one that seeks to create awareness of local events. The notice board styles, posting form factors, and content described above offer a starting point for developers to target their systems; designers should complement this general description with specific knowledge and understanding of their particular users. A digital notice board designed for a chain of coffee shops might for example draw on elements from unscaffolded classifieds and event displays, and would consider the type of clientele, the activities that take place in the shop, and the chain's design aesthetic and brand image.

The design should take into account that users interact with more than one display. In a department store they may see one at the entrance, one in the elevator, and one where they try on some shoes. The design should capitalize on the fact that users will be exposed to a network of displays. If the used technologies allow determining in which order users see the displays the presentation should utilize this, e.g. creating a story within the display content across the physical display network.

\section{B. Respect the Neighborhood Focus of Notice Boards}

Of the thousands of postings we observed across the 29 sites, every one, with almost no exception, was related to the local area or to the community that used the space. The vast majority of these were classifieds relevant to a limited area or notifications for local events. Across the very different styles of notice boards, it is clear that the neighborhood is the audience for and the source of the postings. If a digital notice board is to play the same role, it should be based on postings and ads that have a clear connection to the place or neighborhood, not on centralized ad campaigns. Especially for networked display systems, where technically there is no limitation for the distribution we recommend to design posting procedures in a way that supports locality and to restrict the content to a certain neighborhood. Such mechanisms are most likely a property of the system architecture as well as a part of the actual design.

Note that this is not simply a matter of geographical restriction, but of community identity, even more though when using display networks. The handwritten nature and tear away tabs of many classified postings create an indirect physical connection between advertiser and reader, which may lead to greater intimacy and trust. Further, the aesthetic of a concert poster both communicates the intended audience and is used by that audience to provide a shared group identity. A digital notice board might attempt to capture the direct intimacy of handwritten notes by allowing posters to record short video messages as part of postings. 


\section{Support the Emergent Profiling of a PNA by Owners and Users of the Space}

The different styles of notice boards we observed reflect, among other things, different agendas on the part of the owners of the space. Some are considered decorative or a way to express the identity or support the image of the place, some are appreciated as providing another activity for customers to engage in, increasing the importance of the venue to the community and potentially even attracting customers. Perhaps the most important agenda, however, is in disseminating information the notice board owners have and interest in or sympathy with, such as a library posting notices for classical concerts or an adult learning center ones for trading course books.

Board owners actively use their control to promote all of these priorities. At the same time, we found that in many cases they have difficulty articulating their agendas, and when asked, many were unable to give a good explanation for why they even offered a PNA. Even more importantly, they rely on third-party posters for most of the content. These factors mean that the actual profile of the PNA is usually not predefined, but emerges from the interplay of the interests of the board owners and users.

This goes to the core of reasons why venue owners are willing to offer free advertising space and must be taken very seriously. In a globally networked digital system, the owner of the venue may no longer be the owner of the board and may have a much less direct control of the content that is posted. A digital replacement must give the venue owners overall control over the board profile, and designers must recognize that this is usually not a choice that is made once, but a day-to-day activity. Features could be provided to the managers or owners of each venue allowing them to easily choose which postings to allow or remove (and perhaps learning and automating their patterns over time) and to oversee the overall appearance of the board.

\section{Design for Flexibility of Input, Low Overhead to Post}

Across all locations we found an impressive variety of posts, comprising hand-written notes in various sizes and colors on a set different materials, printed notes enhanced with images or maps, and professionally designed advertisements. We recommend that digital notice boards provide means for preserving this flexibility, supporting:

- Ad-hoc posters: For people (coincidentally) approaching the display a mechanism has to be provided allowing for on-site creation of notes (e.g. predefined templates, standard input devices such as mouse, keyboard, or touch).

- Sophisticated Posters: People preparing content in advance need to be given means to easily transfer it to the board locally, e.g. via a scanner, a USB stick or Bluetooth and in networked settings remotely, e.g.. via a web interface.

- Professional Posters: In a similar way, people distributing professionally designed content (e.g. flyers) need to be granted easy access to the board.

As a result of the flexibility of current approaches we see that the entry barrier is kept low, hence attracting a large amount of posters. We believe that for globally networked display systems the success heavily depends upon speed and ease of use of the content creation mechanisms. 


\section{E. Support Takeaway of Information}

The opportunity to take away information is a crucial prerequisite for the success of classifieds as well as for event promotions. In the design of this mechanism two basic options exist: providing a pointer for the user to take way (e.g. a URL or a phone number) or to provide a copy of the content to take away (e.g. a flyer). Traditional systems use a combination where a pointer is taken away (e.g. by tearing off a piece of paper) and this also contains a minimal summary of the content. In digitally networked systems and considering users equipped with mobile devices many mechanism exist to support users in taking content with them, including users taking photos of the content, to sending this information via SMS or Bluetooth, by providing a QR code, or simply by printing it out. Whereas the primary motivation is to preserve information such as a date, a name, an address, or a telephone number, additional implicit information is embedded with the takeaway information: the number of missing takeaways, e.g. indicates high interest. On digital displays similar information could be provided by displaying the number of poster downloads, or even by restricting the number of possible downloads.

\section{Conclusion and Future Work}

Traditional displays are all around us and interaction with them is a common activity. The communication characteristics we can find in traditional displays are in many ways complementary to the Internet. The importance of using public displays to address a larger number of people with general content has declined with the advent of digital social networks and the World Wide Web. However public displays play a major role in addressing groups of people that can be found in a certain location. We believe that understanding practices in the use of traditional analog displays can provide valuable insights for future generations of globally networked public displays.

Analyzing the observations of the usage of a large number of public displays situated in different context as well as based on follow-up interviews with stakeholders we collected and described various practices related to public displays. In particular we were interested in the various stakeholders and their motivation in the use of public displays. Additionally we investigated how content can be provided and removed and how access control is implemented. Based on our reflection and understanding of the data we suggested a set of design implications for digital display systems.

The central design recommendation is to take the context of a potential display into account: the people likely to pass by, the neighborhood and community in which the display is situated, and the display owners' expectations with regard to content. Beyond this flexible content creation, content posting, and content control are central to allow a broad set of people using it. To increase the effectiveness of posted content it is important that viewer have means to take the information with them - through physical tokens or digital technology.

Currently we are working on an implementation, called Digifieds, which supports practices that are effective in traditional signage systems and that can provide new features for creating rich communication media based on public displays. Digifieds will be deployed as a finalist of the UbiChallenge in Oulu, Finland in summer 2011. 


\section{Acknowledgement}

The research leading to these results has received funding from the European Union Seventh Framework Programme (FP7/2007-2013) under grant agreement no. 244011.

\section{References}

1. Agamanolis, S.: Designing displays for human connectedness. In Public and Situated Displays Social and Interactional Aspects of Shared Display Technologies, Kluwer Academic. pp. 309-334 (2003)

2. Brignull, H., Rogers, Y.: Enticing people to interact with large public displays in public spaces. In Proceedings of INTERACT'03, ACM, Zurich, Switzerland, pp. 17-24 (2003)

3. Churchill, E., Nelson, L., Denoue, L., Girgensohn, A. The Plasma Poster Network: Posting Multimedia Content in Public Places. In Proc. INTERACT'03, Zürich, Switzerland, 2003.

4. Churchill, E. F., Nelson, L., Denoue, L.: Multimedia fliers: Information sharing with digital community bulletin boards. In Communities and technologies, Kluwer Academic, Deventer, Netherlands, pp. 97-117 (2003)

5. Churchill, E. F., Nelsen, L., Denoue, L., Helfman, J., Murphy, P.: Sharing multimedia content with interactive public displays: a case study. In Proceedings of the DIS'04, ACM, Cambridge, MA, USA, pp. 7-16 (2004)

6. Ferscha, A., Vogl, S.: Pervasive Web Access via Public Communication Walls. In Proceedings of Pervasive Computing'02, Springer, Zurich, Switzerland, pp. 84-97 (2002)

7. Greenberg, S., Boyle, M., Laberge, J.: PDAs and shared public displays: Making personal information public, and public information personal. In Personal and Ubiquitous Computing'99, Springer, pp. 54-64 (1999)

8. Huang E. M., Koster, A., Borchers, J.: Overcoming assumptions and uncovering practices: When does the public really look at public displays?. In Pervasive Computing '08, Springer, Sydney, Australia, pp. 228-243 (2008)

9. Huang E. M., Mynatt, E. D., Trimble, J. P.: When design just isn't enough: the unanticipated challenges of the real world for large collaborative displays. In Personal and Ubiquitous Computing, Springer, pp. 537-547 (2007).

10. McCarthy, J. F., Farnham, S. D., Patel, Y., Ahuja, S. Norman, D., Hazlewood, W. R. Lind, J.: Supporting community in third places with situated social software. In Proceedings of C\&T'09, ACM, University Park, PA, USA, pp. 225-234 (2009)

11. Michelis, D., Müller, J. 2010. The Audience Funnel. In: International Journal of HCI

12. Nakamura, M. A.: Creating a new channel for campus communication. In Proceedings of ACM SIGUCCS'04, ACM, Baltimore, MD, USA, pp. 56-59 (2004)

13. Redhead, F. Brereton, M.: A qualitative analysis of local community communications. In Proceedings of OZCHI '06, ACM, Sydney, Australia, pp. 361 - 364 (2006)

14. Redhead, F., Brereton, M.: Designing interaction for local communications: an urban screen study. In Proceedings of INTERACT'09, ACM, Uppsala, pp. 24-28 (2009)

15. Storz, O. Friday, A. Davies, N., Finney, J., Sas, C. and Sheridan, J.: Public Ubiquitous Computing Systems: Lessons from the e-Campus Display Deployments. In IEEE Pervasive Computing, 05(3): pp. 40--47 (2006)

16. Taylor, N., Cheverst, K.: Exploring the Use of Non-Digital Situated Displays in a Rural Community. In: Workshop on Public and Situated Displays to Support Communities (2008)

17. Taylor, N., Cheverst, K., Fitton, D., Race, N. J., Rouncefield, M., and Graham, C.: Probing communities: study of a village photo display. In Proceedings of OZCHI '07, pp. 17-24.

18. Weiser, M.: The computer for the 21st century. In Scientific American, pp. 65-75 (1991) 